\title{
Sensor-Fusion methodology for reducing product quality variation
}

\author{
J. Mou \\ Department of I\&MSE \\ Arizona State University \\ Tempe, AZ 85287 \\ S. D. Jones \\ R. J. Furness \\ Manufacturing Systems Department \\ Ford Scientific Research Laboratory \\ Dearborn, MI 48121
}

\begin{abstract}
As competitive pressures demand higher levels of productivity and product quality, the accuracy and reliability of products remains important but becomes increasingly more difficult to achieve. The dimensional inaccuracy of any manufactured part is a combined effect of several degradation factors including process related errors, machine related errors, fixturing related errors, material property variation, and dimensional variation of the incoming parts or raw material. The inspection processes have become an integral part of the manufacturing processes and form the metrology anchor to determine the quality of manufactured products and to certify the performance of manufacturing processes. However, there still remains a lack of a comprehensive methodology, to identify and assess the contribution of those factors that cause variation in product quality, based solely on inspection data.

The objective of this study is to advance the technology, precision, and functionality of machining centers by developing a sensor-fusion methodology that integrates pre-process, on-machine, and post-process measurement and analysis technology to cost-effectively enhance the accuracy and precision of both manufacturing and inspection processes. The primary effort is directed at the development of a sensor fusion methodology that captures the part error signature. Non-traditional sensory data analysis algorithms will also be developed to identify


the contribution of each degradation factor, based solely on the error signature. Finally, an adaptive machining process control algorithms will be derived to reduce product quality variation.

\section{Keywords}

Sensor-fusion, Error modeling, Error correction, Product variation reduction.

\section{INTRODUCTION}

A significant portion of the cost in small batch manufacturing results from verifying workpiece form, finish, and dimensions, and then scrapping or reworking rejected parts. Historically, inspection data was used to determine whether or not a manufactured part met specifications. If it did not, it was reworked or rejected. Recently, significant progress has been made in determining the quality of manufacturing products and certifying the performance of manufacturing process (ANSI, 1985, 1990, 1992, 1994). Inspection procedures are commonly used to identify the resultant variation in product quality. Now inspection devices like coordinate measuring machines (CMMs) can provide data about how far a part is in or out of tolerance. This data is now being used to make manual adjustments to machining offsets and process parameters.

Ideally, the same inspection data should also be used for controlling and reducing the process variation. In reality, it is a very difficult task due to the lack of methodology that leads to the determination of the actual contribution of each degradation factor in affecting the product quality variation. In anticipation of the future of manufacturing technology, when machine tools are equipped with openarchitecture controls and advanced sensing technology, the information from these sensors must be processed and used to make adjustments at the machine level. Therefore, there is a critical need to develop such methodology to identify the contribution of each degradation factor by innovatively interpreting the inspection results. In order for this vision to be realized, it is necessary to develop analytical models between dimensional measurement sensor data and the factors that result in increased process variations. These models do not yet exist, but can be developed from in-depth analysis encompassing advances in machine tool metrology, dimensional metrology, and process control.

To achieve this goal, sensor-fusion technology and non-traditional inspection analysis techniques are needed to identify the signature and contribution of various factors that cause variation of product quality in dimension [Swyt, 1992], form (Menq, et. al., 1990), surface finish (Shin, et. al., 1995), and sub-surface material properties (Nguyen, et. al., 1995). Meanwhile, part features produced by different machines with specific manufacturing processes will have different signatures. Therefore, there is a need to develop algorithms to assist the process of selecting the proper sensors, sampling procedures, and the sampling size for each feature (Woo \& Liang, 1993; Lee \& Mou, 1996). To ensure the quality and reliability of inspection process, measurement uncertainty and error needs to be addressed for all data acquisition activities (Hocken, 1995). 
The efforts of this study are to develop a sensor-fusion methodology that integrates the aforementioned technologies to capture the signature of part quality variation. Innovative sensory data analysis algorithms also need to be developed to identify the contribution of each degradation factor, solely based on its individual error signature. Further, adaptive machining process control algorithms need to be derived to reduce product quality variation. The result could significantly advance the accuracy and functionality of automated machinery. This study is trying to provide a means for integrating more sensing and analyzing capabilities at the machine level, and to generate methods for deriving information describing machine, process, and fixturing related errors from various sensor-fusion techniques. From gauging the part where it is machined, to post-process fixture gauging that can be tied into on-machine gauging to audit the machine, the methodology developed under this study could reduce cost and dramatically improve manufacturing performance.

A major goal is to make high quality parts with the least amount of scrap. Other objectives of this project are to integrate data acquisition and evaluation functions with machining functions, provide on-line quality analysis and feedback, and develop methods of using sensor data to perform real-time adaptive sensor feedback process controls to reduce overall product variations. Since more accurate and on-time product monitoring and control will be achieved, the methodology will improve part-to-part repeatability, increase flexibility, reduce cycle time, and increase the accuracy of the machined features. The resulting technology advancement and implementation will benefit industry in reducing production costs and increasing competitiveness.

Given that the research is successfully executed, the cause and effect relationship of product and process variation resulting from machine related errors, process related errors, and fixturing related errors can be identified and then minimized. Meanwhile, the results of developing non-traditional measurement procedures and sampling strategies will advance the technology in extracting information that truly represents the actual dimensions and the form of measured features. Therefore, the accuracy and reliability of both manufacturing and inspection processes can be improved dramatically. The developed methodology that integrates sensor-fusion technologies could significantly facilitate the implementation of the error modeling and correction techniques in performance monitoring and tracking for manufacturing processes. The developed algorithms can then be implemented on a PC-based open-architecture controller (Wright, et. al., 1996) to receive information from various sensors, assess the status of the process, determine the proper action, and deliver the command to actuators for task execution. This will enhance a CNC machine's capability to produce workpieces within the imposed dimension tolerances.

This project has proceeded in several dimensions in parallel. In the following sections, only selected major elements are discussed in detail. 


\section{METHODOLOGY}

Multi-axis machines are typically composed of a sequence of elements or links connected by joints, providing either rotational or translational motion. By means of a kinematic analysis, each link and joint can be modeled as a link transformation matrix and a joint constraint matrix, respectively. There are two types of kinematic relationship between two adjacent components: stationary transformation and motion transformation. Machine errors result from both imperfect stationary configuration and imperfect motion of those components. The spatial displacement error of the cutting tool or end effector is affected by the translational and rotational errors in each individual axis.

A kinematics-based analytical model is derived to describe the degradation effect of machine error (i.e., spindle growth, linear displacement error, straightness error, etc.) on a part's dimensional and form accuracy. A generic model generator has been developed to enable analysts to systematically formulate an appropriate error model that establishes the relationship between the component errors and the observed volumetric errors for various machine configurations. An explicit, parametrically represented generic function describing the machine error is therefore obtained. For error compensation, the corrective matrix and modified input command is also derived using this generic function. The generic model generator is based upon the concepts of functional variation, rigid body kinematics, and homogeneous transformation.

To include the physical imperfections of a multi-axis machine, the link transformation matrix, $L_{i}$, is used to describe the relative position of two coordinate systems on an inaccurate link (Mou \& Liu, 1992). The joint constraint matrix, $J_{i}$, is used to describe the relative motion of two coordinate systems along an imperfect prismatic or revolute joint. A homogeneous transformation matrix, $A_{i}$, for an inaccurate positioning element is then expressed as $A_{i}=L_{i} * J_{i}$. Given any multi-axis machine with an arbitrary combination of prismatic and revolute joints, this homogeneous transformation matrix is used to build a mathematical model describing the resultant positioning errors as follows:

$$
\left[\bar{e}_{w}\right]=[T-I]\left[\bar{r}_{i d e a l}\right]=\left[\left[\prod_{i=1}^{n}\left[A_{i}\right]\right]-[I]\right]\left[\bar{r}_{i d e a l}\right]
$$

The input to the error model includes the nominal tool positions, temperature profile of the machine structure, and machine configuration constants. The output of the error model is the predicted machine tool error within the designated workspace.

The major advantage of this modeling approach is that a kinematics based model provides a systematic way to model, estimate, and compensate for the positioning error of a multi-axis machine. By using the inverse kinematics technique, the contribution of each error component in each axis can be systematically determined. The information is subsequently used to calculate the error compensation signal for each axis to correct machine error. For machine error 
correction, the relationship between the error compensation signal and the corrected position vector of the tool tip is expressed as

$$
\left[\bar{r}_{\text {compensated }}\right]=[T]\left[\bar{r}_{\text {tool }}+\bar{e}_{c}\right]
$$

Rearranging the matrices, the error compensation signal is then obtained as

$$
\left[\bar{e}_{c}\right]=\left[\boldsymbol{T}^{-1}-I\right]\left[\bar{r}_{\text {tool }}\right]
$$

Commercially available software (for example, MAPLE or MATHEMATICA) with symbolic manipulation functions are used in developing the proposed generic model generator. Boolean algebra are used to eliminate the cross over terms in stepwise fashion and, thus, to reduce the complexity involved in the derivation of the model. A window based graphical interface is currently been developed to help the analysts graphically formulate the manipulating sequence of transformation matrices for various machine configurations. Once the sequence for matrix manipulation is determined, the developed generic function can then automatically generate the corresponding resultant transformation matrices for both machine error modeling and compensation.

To fully utilize the derived generic model for machining system performance assessment, the appropriate sensors and sampling techniques for capturing the signature of various degradation factors need to be determined. In this project, touch-trigger probes are used in on-machine gauging and post-process inspection to determine the dimensional accuracy of manufactured parts. Analog scanning probes are used in on-machine gauging and post-process inspection to determine the form accuracy of manufactured parts. In a second phase of this project, a video probe will be used to inspect surface texture, burr formation, chip breaking, and tool wear. The inspection data collected from different sensors not only provide information on part quality variation but also on machine performance variation. For example, by comparing the data obtained from on-machine measurement and post-process inspection, the variation in machine accuracy at various thermal states can also be captured. The degradation effect of process related errors (i.e., tool deflection, tool wear, cutting force related workpiece deformation, chatter, etc.) on a part's dimensional accuracy, form accuracy, surface texture, and surface finishing can also be characterized.

For any monitoring or inspection activity, sampling methods used in data collection will affect the precision of the measurement results. Because the analysis model and algorithms consider only those data points that are sampled, any valuable information represented by the unsampled points will be overlooked. Therefore, sampling strategies should be carefully considered during the data collection. From a statistical viewpoint, sampling methods and their designs are the keys to permit valid inferences about any inspection result. Each set of measured data contains a certain amount of information about the inspected object. The quantity of information obtained in the sample depends on the number of data points sampled and on the amount of variation in the data. 
Since different features produced by a particular type of machine or machining process will have different signatures, algorithms need to be developed to assist the process of selecting the proper sampling strategy and the sampling size for various features. A feature-based sampling strategy (Lee \& Mou, 1996), based on the Hammersley sequence (Woo \& Liang, 1993) and the stratified sampling method, is implemented to locate sampling points on different geometric features. The design of the sampling strategies and the sample size determine the quantity of information in the sample pertinent to a population parameter, provided that accurate measurements are obtained on each sampled element. Usually, the best design for a given problem is the one that provides the necessary precision in terms of a bound on the error of estimation for a minimum cost. Statistical methods are used to determine the proper sampling rate. Meanwhile, the number of specified measuring points and its relation to time, cost, and accuracy is currently been investigated.

For all data collection activities, measurement uncertainty needs to be understood and minimized. Since various sensors will be used in this sensor-fusion based projects, the measurement uncertainty assessment becomes more complex especially when machine errors are present. Case studies are conducted to investigate the effect of both individual and confounded measurement uncertainties resulting from sensors and measurement procedures. For example, in most inspection operations using a CMM, the data acquisition process is conducted while controlling each axis either individually or simultaneously. Nevertheless, for all operation modes, the errors in each axis will affect the measurement accuracy. The degradation effect will vary as a function of the characteristics of the machine error and the geometric feature of the measured artifact. As shown in Figure 1, when a designated nominal point $N=\left(x_{N}, y_{N}\right)$ on the workpiece is measured, the measuring process can be divided into two stages. In the first stage, the probe is quickly moved to a temporary point $N_{I}=\left(x_{N I}, y_{N D}\right)$ which is a nominal point relative to $N$. Usually, for every measuring machine, the distance between $N$ and $N_{l}$ is the default or user selected approach distance $d$. From point $N_{l}$, the probe is then moved slowly toward the designated measurement point $N$ in the second stage to assure an accurate measurement. 


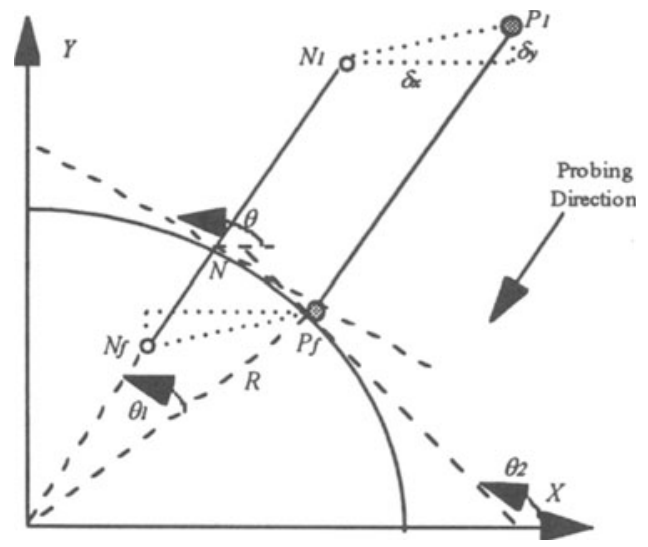

Figure 1 Potential error in measuring a circular feature along the normal direction.

If there are machine errors $\left(\delta_{x}\right.$ and $\left.\delta_{y}\right)$ in both $x$-axis and y-axis, the probe may not be exactly located at the point $N_{1}$, but will be located at $P_{l}$ instead. Then, the probe will be moved along the designated approach direction that is usually parallel to $x-$ axis, y-axis, or normal to the feature surface until a contact occurs. However, due to machine related error, the machine controller cannot detect the error and may command the probe to move along a path slightly different from the designated path. The offset of the new path is a function of machine errors. Depending on the relative measurement direction and geometric characteristics of the measured feature, the inspection data will be contaminated by different types of confounded uncertainties. For example, when the probe finally contacts the measured feature at point $P_{f}$, the machine controller may interpret the point $P_{f}$ as point $N_{f}$ based on the distance the probe traveled. Therefore, the uncertainties result from the machine errors and the geometry characteristics of the measured feature, as well as the dimensional imperfection of the measured feature.

Generic analytical models and computational algorithms are derived to assess the confounded effect of various types of uncertainties occurring in common measurement procedures. To improve the effectiveness of machine performance calibration and on-machine inspection process, analytical methods are derived to assess the confounded effect of machine errors, geometric characteristics of measured features, and dimensional imperfection of measured features. The development of the analytical models and computational algorithms is carried out in several sequential steps. In the first step, the uncertainty effect of an imperfect measuring device is considered while assuming the measured features have perfect dimensions and forms. Therefore, the confound effect is a joint product of the equipment imperfection and geometric characteristics of the measured features. In the second step, the uncertainty effect of an imperfect measuring device, the geometric characteristics, and dimensional imperfection of the measured features are considered. 
After analytical models are derived for various uncertainty effects, new measurement methods and algorithms for uncertainty decoupling and reduction need to be developed. The effectiveness and the limitation of the derived uncertainty models, the uncertainty decoupling and reduction algorithms need to be investigated. Simulation and experimental analysis are then conducted to validate the effectiveness of the derived method (Lee, et. al., 1997a, b).

Since the dimensions and the form of each manufactured feature always differ from the nominal dimensions and the perfect form, most existing fitting algorithms cannot provide the information needed for part error signature characterization. For part error signature characterization, we need to know the actual dimensions and the geometric form of the manufactured feature instead of the substitute features of perfect geometric forms. Therefore, an alternative way of sampling and analyzing data is needed to extract feature parameters that truly represent the actual dimensions and the form of measured features.

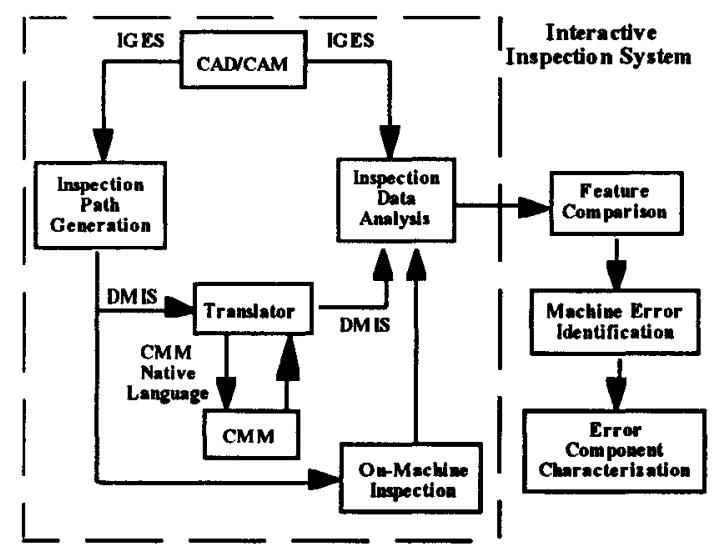

Figure 2 Interactive inspection system and part error characterization.

An interactive inspection algorithm, as shown in Figure 2 (Mou, et. al., 1995), is developed to compare the inspection data obtained from both on-machine sensing and post-process inspection to identify systematic dimensional and form errors. The results of the proposed interactive inspection algorithm will be used to i) compare the data obtained from on-machine and post-process inspections, ii) decouple the machine related error and process related error, and iii) identify the contribution of each error component.

To characterize the machine related error, the geometric inaccuracy must be related to the error components that compose the volumetric machine error. A feature-based analysis method is developed to decompose the geometry of any part into a collection of basic geometric features (such as cylinders, cones, circles, hemispheres, planes, etc.) as defined in Dimension Measurement Interface Standard (DMIS, 1990). A feature-based comparison method is also developed to identify and then decouple the effect of machine related and process related errors. 
The same inspection information is used to verify the effectiveness of the onmachine inspection and machine error characterization.

After the degradation effect of machine error and process error are identified and decoupled, the inspection analysis results can then be used to extract information regarding error resulted from tool wear, tool deflection, chatter, and fixture/workpiece deformation. The dimensional inaccuracies of different features can be used to extract information regarding the systematic machine error and process error. For a particular type of machining process, the potential error sources for each feature can be categorized and used to derive a set of diagnostic equations to identify the individual degradation factor. As the contribution of each degradation factor to part quality is determined, the results can be used by the adaptive error correction system to iteratively improve the machine performance.

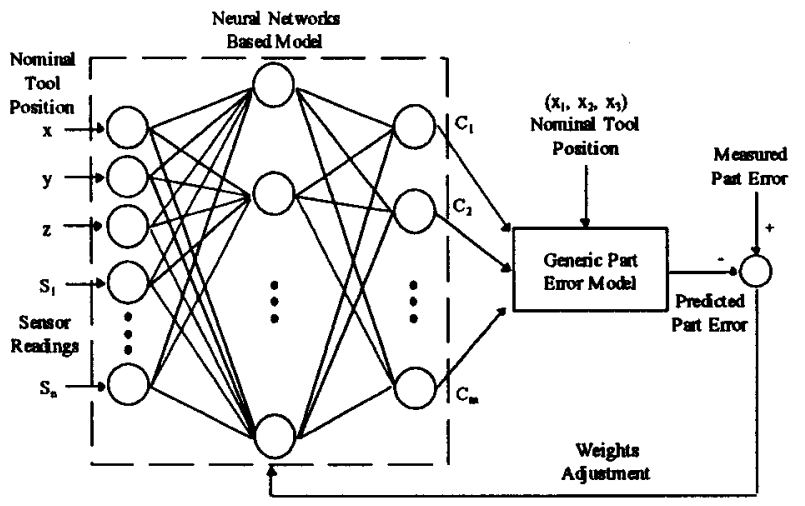

Figure 3 Integrated error modeling system for rapid characterization of error signature.

In this project, as shown in Figure 3, an innovative approach is also developed to simplify the parameter estimation and validation phase by interactively integrating neural networks and those derived generic models (Mou, 1997). The developed integrated system can track the time-varying part quality variation by adapting current inspection results obtained from various sensors. The goal is to develop a fast characterization process to build a model for characterizing the variation of the production process by adopting a sensor-fusion approach.

In deriving the coefficients of the proposed integrated system, there is no measured data available for direct comparison with the neural network output. Instead, the difference between the predicted value from generic models and the measured value is used to train the neural network. Therefore, a new training algorithm is developed for this integrated error modeling system. For data-adaptive updating purposes, the gradient-descent direction method is adopted in deriving the training algorithm. Issues related to convergence rate, stability, training rate, and number of hidden nodes are also investigated.

A methodology is currently being developed for using on-machine inspection techniques to identify the fixturing related error in terms of offset in part datum co- 
ordinates and the deformation of the workpiece and fixturing components due to the clamping forces and clamping sequence. The goal is to develop a method for shop floor personnel to conduct a quick checking, after clamping forces are applied, on the deformation of critical machining surfaces.

An adaptive algorithm is currently being derived for iteratively identifying the contribution of each degradation factor. The output of the adaptive algorithm will be merged with the cutting force prediction model, next generation inspection system (Jones, et. al., 1996), and the plug-and-play software development environment of Figure 4 (Furness, et.al., 1996) that is currently under development by Ford's research engineers to determine the proper setting of NC tool path, tooling, fixturing, and machining parameters to minimize the variation in part quality and production cost.

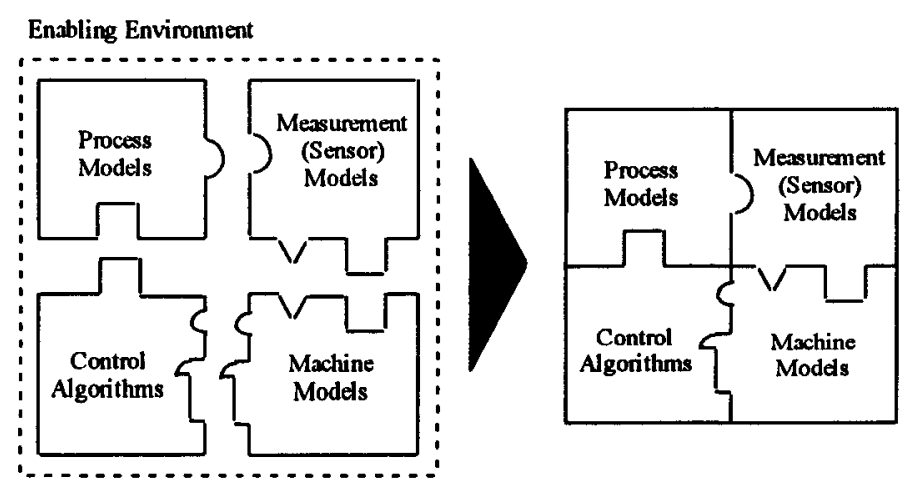

Figure 4 Plug-and-play software development environment.

A recursive fine-tuning algorithm, as shown in Figure 5 (Mou, et. al., 1995a, b), is also being derived to iteratively refine the model. The objective is to investigate a concept for developing an adaptive error correction system that integrates the methods and algorithms proposed in previous sections to facilitate the following procedures: (1) identification of the systematic degradation factors, (2) development of the multi-variable model for part error estimation and compensation, (3) detection of the residual systematic errors that are not considered by the model using information obtained from both on-machine sensing and post-process inspection processes, (4) identification and characterization of the contribution of each residual systematic degradation factor, and (5) fine-tuning the model parameter for more accurate part error estimation and compensation. 


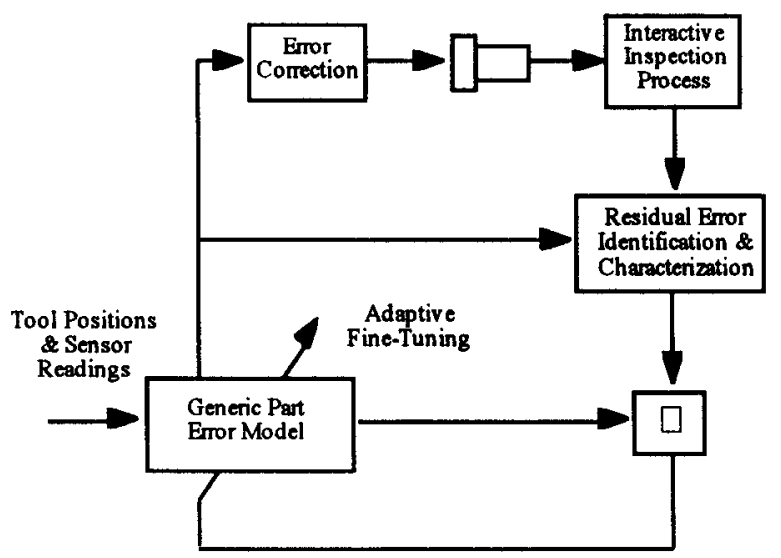

Figure 5 Recursively fine-tuning the model for part error estimation and compensation.

By using the feature-based analysis method discussed in the previous section, the contribution of the degradation factor can be iteratively monitored and determined. As the manufacturing process proceeds, this process is iteratively executed as more information regarding the part error signature becomes available. Therefore, the inference space of the proposed integrated part error modeling system is continuously updated.

\section{CONCLUSION}

This research is motivated by the need to develop innovative and cost-effective methods to advance the machine technology for precision and agile manufacturing applications. The impacts of the research can be summarized as follows.

The results of the research will significantly advance the accuracy and functionality of manufacturing and measuring equipment. This research effort is devoted to the improvement of the precision of machining centers through advances in dimensional measurement and analysis techniques. This research provides methods for integrating more sensing and analysis capabilities at the machine level. The developed sensor-fusion methodology provides the mechanism for determining the degradation effect of machine error, process error, and fixturing error on part quality variation. The derivation of uncertainty models for sensor setting and measuring processes will significantly contribute to the development of novel techniques for data sampling and analysis, and thus will have a significant impact on precision manufacturing.

The results of advancing the on-machine and post-process sensing and analysis techniques will have significant impact on research into precision and agile engineering applications. The research results can be used to identify, decouple, and then minimize confounded measurement uncertainties. The results of developing innovative measurement procedures and sampling strategies will 
advance the technology in extracting information that truly represents the actual dimensions and the form of measured features. Therefore, the quality and reliability of the inspection data can be improved dramatically and a more accurate and meaningful conclusion can be drawn.

The development of an adaptive error modeling system with sensor-fusion technologies will significantly facilitate the implementation of the error modeling and correction techniques for various manufacturing equipment. The adaptive error modeling system can be easily implemented on a PC based open-architecture controller to receive information from various sensors, assess the status of the process, determine the proper action, and deliver the command to actuators for task execution. This will enhance machine capabilities to produce workpieces within the imposed dimension tolerances.

\section{REFERENCES}

ANSI/ASME PTC 19.1-1985, 1985, Measuring Uncertainty, ASME, New York.

ANSI/ASME B89.1.12M-1990, 1990, Methods for Performance Evaluation of Coordinate Measuring Machines, ASME, New York.

ANSI/ASME B5.54-1992, 1992, Methods for Performance Evaluation of Computer Numerically Controlled Machining Centers, ASME, New York.

ANSI/ASME Y14.5M-1994, 1994, Dimensional and Tolerancing, ASME, New York.

DMIS, 1990, "DMIS 2.1," Specification CAM-I Standard 101, CAM-I, Inc., Arlington, TX.

Furness, R.J., Jones, S.D., Rankin, J.S., Muth, M.J., Wei, K.C., and Copper, J.C., 1996, "Control System Requirements to Support Intelligent Sensor-Based Manufacturing," Proceedings of the 1996 IPC Conference and Exposition, pp.55-62.

Hocken, R.J., 1995, "Measurement Integration," in Coordinate Measuring Machines and Systems, edited by Bosch, J.A., Marcel Dekker, Inc., New York.

Jones, S.D., Furness, R.J., Muth, M.J., and Rankin, J.S., 1996, "Agile Metrology:

The Next Generation of Measuring Machines," Proceedings of the 1996 IPC Conference and Exposition, pp.97-102.

Lee, G. and J. Mou, 1996, "Design the Sampling Strategy for Dimensional Measurement of Geometric Features using Coordinate Measuring Machine," Proceedings of Japan-U.S.A. Symposium on Flexible Automation, pp.1193-1200.

Lee, G., J. Mou, and Y. Shen, 1997a, "An Analytical Assessment of Measurement Uncertainty in Precision Inspection and Machine Calibration," International Journal of Machine Tool and Manufacture, Vol. 37, No. 3, pp. 263-276.

Lee, G., J. Mou, and Y. Shen, 1997b, "Decoupling the Confounded Effect of Machine Error and Geometric Characteristics of Artifacts in Precision Measurement and Machine Calibration," submitted to ASME Transactions on Journal of Manufacturing Science and Engineering. 
Menq, C.-H., Yau, H.-T., Lai, G.-Y., and Miller R.A., 1990, "Statistical Evaluation of Form Tolerances Using Discrete Measurement Data," Proceedings of ASME Winter Annual Meeting, pp.135-149.

Mou, J, and Liu, C.R., 1992, "A Method for Enhancing the Accuracy of CNC Machine Tools for On-Machine Inspection," Journal of Manufacturing Systems, Vol.11, No.4, pp.229-237.

Mou, J., Donmez, M.A., and Cetinkunt, S., 1995a, "An Adaptive Methodology Using Features-Based Analysis Techniques for Machine Performance Improvement: Part I - Theory Derivation," ASME Transactions on Journal of Engineering for Industry, Vol.117, No.4, pp.584-590.

Mou, J., Donmez, M.A., and Cetinkunt, S., 1995b, "An Adaptive Methodology Using Features-Based Analysis Techniques for Machine Performance Improvement: Part II - Experimental Verification," ASME Transactions on Journal of Engineering for Industry, Vol.117, No.4, pp.591-600.

Mou, J., 1997, "A Method of Using Neural Network and Inverse Kinematics for Machine Tool Error Estimation and Correction," ASME Transactions on Journal of Manufacturing Science and Engineering, Vol.119, No.2, pp.247254.

Nguyen, T.L., Furgason, E.S., and Shin, Y.C., 1995, "Feasibility Assessment of Residual Stress Measurement by an Ultrasonic Technique," Transactions of the North American Manufacturing Research Institution of SME, pp.287-292.

Shin, Y.C., Oh, S.J., and Coker, S.A., 1995, “ Surface Roughness Measurement by Ultrasonic Sensing for In-Process Monitoring," ASME Transactions on Journal of Engineering for Industry, Vol.117, No.3, pp.439-447.

Swyt, D.A., 1992, Challenges to NIST in Dimensional Metrology: The Impact of Tightening Tolerances in the U.S. Discrete-Part Manufacturing Industry, NISTIR 4757, National Institute of Standards and Technology, MD.

Woo, T.C., and Liang, R., 1993, "Dimensional Measurement of Surfaces and Their Sampling," Computer-Aided Design, Vol.25, No.4, pp.233-239.

Wright, P., Schofield, S., and Wang F.-C., "Open Architecture Control For Machine Tools," Proceedings of 13th Symposium on Engineering Applications of Mechanics: Manufacturing Science and Engineering of 1996 CSME Forum, pp.1-17. 